\title{
Sinonasal IgG4-RD: Case Report and Systematic Review
}

\author{
Caleb Wilson ${ }^{1}$, Benjamin Brownlee ${ }^{2}$, Edward El Rassi ${ }^{3}$, and Kibwei McKinney ${ }^{3}$ \\ ${ }^{1}$ University of Oklahoma Health Science Center \\ ${ }^{2}$ University of Oklahoma Health Sciences Center \\ ${ }^{3}$ The University of Oklahoma Health Sciences Center
}

September 24, 2021

\begin{abstract}
Immunoglobin G4 - related disease (IgG4-RD) is a chronic fibro-inflammatory condition that presents as a single or multiple tumefactive lesions affecting virtually any organ system. Here we report a case of recurrent sinonasal IgG4-RD and review the literature of this evolving entity.
\end{abstract}

\section{INTRODUCTION}

Immunoglobin G4-related disease (IgG4-RD) is a chronic fibro-inflammatory disease that presents as one or multiple tumefactive lesions affecting virtually any organ system. It was first described in 2001 by Hamano et al. in patients with autoimmune pancreatitis and elevated serum IgG4 levels. ${ }^{1}$ In 2011, an international consensus on the diagnosis of IgG4-RD was developed, which requires histologic evidence of IgG4 positivity in addition to other hallmark features including lymphoplasmacytic infiltration, storiform fibrosis, obliterative phlebitis, and mild to moderate tissue eosinophilia. ${ }^{2}$ The exact etiology of IgG4-RD is poorly understood, but recent studies have shown a clear role of type 2 helper $\mathrm{T}$ cells and $\mathrm{T}$ regulatory cells. IgG4 itself seems to be a protective response to the inappropriate immune activation, rather than pathologic. ${ }^{3}$

While first described in the pancreas, manifestations of the disease have been described throughout the body, including the lacrimal glands, submandibular glands (Kuttner tumor), orbit, lymph nodes, thyroid, biliary tract, liver, kidney, retroperitoneum, lungs and even aorta. Mulholland et al. ${ }^{4}$ demonstrated that the condition shows a predilection for the head and neck, with the orbit or salivary glands being the most frequently involved. Although the head and neck region is the second most common location of involvement for IgG4-RD, the paranasal sinuses are rarely affected, and the first sinonasal case was described in 2009 by Ishida et al. ${ }^{5}$ In 2015, Song et al. ${ }^{6}$ published the first sinonasal-specific IgG4-RD literature review in which it was described as an emerging entity. At that time, only 8 cases had been described in the literature that met current diagnostic criteria, but since that review many other cases have been reported. ${ }^{2}$ This report will add to the existing data on sinonasal IgG4-RD with the present case, which describes the longest-known course of this disease a single patient, spanning over 22 years. A comprehensive review of the literature on this topic will then be provided. Finally, strategies for the early recognition and diagnosis of IgG4-RD will be discussed and the efficacy of sinus-specific treatment modalities will be reviewed.

\section{METHODS}

A comprehensive literature review using PubMed and Web of Science was performed through February 2021 following Preferred Reporting Items for Systematic Reviews and Meta-Analyses (PRISMA) guidelines. Articles related to IgG4-related disease with sinonasal involvment were identified using the search terms "IgG4-related disease," "sinonasal IgG4," and "sinus IgG4." Upon removing duplicates, titles and abstracts were screened for relevant articles. English, full-text case reports and series were included. Non-English, 
abstract-only text, and review articles were excluded. Studies that did not meet histopathological diagnostic criteria for IgG4-related disease as described by Deshpande et al. ${ }^{2}$ were further excluded. Ultimately, 20 references meeting the selection criteria were included. ${ }^{5-24}$ Epidemiology, presentations, imaging characteristics, locations of involvement, IgG4 serum and histopathologic levels, treatments rendered, and follow-up of all 31 cases (when available) were extracted and summarized.

\section{CASE REPORT}

This is the case of a 72-year-old male whose disease course began with the development of a spontaneous cerebrospinal fluid (CSF) leak in 1999. His early clinical course from the onset of symptoms up until 2012 was described by $\mathrm{Hu}$ et. al. ${ }^{25}$ in the Annals of Allergy, Asthma, and Immunology in 2013, which this manuscript will briefly recount. It is the goal of this paper to present his disease course following Rituximab infusion, as well as the long-term follow up between 2013 and 2021. This patient's disease initially manifested with multiple systemic features, including pulmonary emboli, xerostomia, and intermittent double vision; however, the early stage of disease is not the focus of this paper and is therefore is not extensively discussed.

In 1999, at the age of 50 years, the patient presented with CSF rhinorrhea and was incidentally noted to have left ethmoid sinusitis on CT. He underwent a craniotomy for repair, and the surgeon noted that that the dura had a $2 \mathrm{~mm}$ defect in the area of the left cribriform plate. The area of infection in the left ethmoid was not addressed. His repair was completed without complication and he remained asymptomatic for over a decade.

He presented to another otolaryngologist in 2012 following a 12-month history of recurrent acute sinusitis. Imaging at that time, including CT and MRI revealed opacification of the left-sided paranasal sinuses, hyperostotic bone, and a contrast-enhancing lesion of the left ethmoid cavity that was concerning for malignancy. Endoscopic sinus surgery was performed shortly thereafter, revealing diffuse mucosal inflammation without evidence of a discrete mass lesion. Multiple biopsy specimens were sent intraoperatively. The final pathology revealed a lymphoplasmacytic infiltrate with areas of fibrosis in a storiform pattern, as well as plasma cells that stained positive for IgG4. His IgG4/IgG ratio was $>80 \%$ in the ethmoid specimen, $>40 \%$ in tissue from the maxillary sinus, and serum IgG4 was within normal limits $(88 \mathrm{mg} / \mathrm{dL}$; normal $<121 \mathrm{mg} / \mathrm{dL})$. He was initially diagnosed with inflammatory pseudotumor; however, the report was addended several weeks later, and the diagnosis of IgG4-RD was subsequently made.

Shortly after diagnosis, he was referred to an IgG4-RD specialist in Boston, MA and underwent two consecutive Rituximab infusions. One month after the infusions, his IgG4 level was reduced to $55 \mathrm{mg} / \mathrm{dL}$. The patient noted symptomatic relief, specifically a reduction in sinus pressure and pain, and in-office nasal endoscopy confirmed a marked improvement in the degree mucosal inflammation. He remained asymptomatic for over a year but his symptoms returned, once again manifesting with left-sided sinus pressure, pain, and purulent rhinorrhea.

Conservative treatment with Budesonide irrigation and culture-directed antibiotics failed to adequately manage his symptoms. A post-treatment CT scan revealed opacification of residual left-sided ethmoid air cells, hyperostotic bone and mucosal thickening within the left maxillary sinus. He underwent revision endoscopic sinus surgery, including left maxillary antrostomy, total ethmoidectomy, and frontal sinus exploration, which was complicated by a CSF leak from the left lateral lamella of the cribiform plate. This was repaired with a free mucosal graft from the left middle turbinate. His surgical pathology showed an IgG4/IgG ratio was $>70 \%$.

Over the following year, his chronic rhinosinusitis (CRS) symptoms were successfully managed with nasal saline irrigations and intermittent use of oral steroids and culture-directed antibiotics for disease exacerbations. The frequency of infections and the number of antibiotics prescribed began to increase over the following year, with worsening polypoid edema in the left maxillary sinus on follow-up endoscopy. A third Rituximab infusion was performed in 2016, and at that time his IgG4 level was $45 \mathrm{mg} / \mathrm{dL}$. Unlike his prior infusions, his symptoms did not improve as dramatically, and he had four sinus infections requiring antibiotics over the ensuing 12 months. A left maxillary mega-antrostomy was performed in October 2016, and 
again the pathologic evaluation of the tissue supports the diagnosis of IgG4-RD. Following surgery, he was again started on Budesonide irrigations which he continued daily for over a year, after which he transitioned to irrigations with buffered saline alone. Over the following years, he has remained mostly asymptomatic and only required intermittent culture-directed antibiotics for sinus infections. At his most recent clinic appointment in December of 2020, his Sino-Nasal Outcome Test-22 (SNOT-22) score was three, and he had not experienced a sinus infection for over a year.

\section{DISCUSSION}

IgG4-RD is a rare fibro-inflammatory condition characterized by tumefactive lesions that can arise in multiple different sites. First described in 2001 by Hamano et $\mathrm{al}^{1}$, this disease process has several areas that remain to be understood, including how to recognize it. IgG4-RD is difficult to diagnose because it can mimic a variety of autoimmune and neoplastic diseases, and it also has non-specific radiologic features. A patient with primarily or exclusively sinonasal involvement may be uniquely challenging to diagnose, especially if they present with classic features of CRS.

\section{Location}

While it is common for IgG4-RD to involve multiple different organ systems at presentation, it seems that sinonasal IgG4-RD is often limited to local disease or only head and neck involvement. Of the 31 cases in this review, $14(45 \%)$ had extra-sinonasal involvement, most of which involved the salivary glands or orbit. Only 2 of these cases involved organs outside of the head and neck, and the present case is the third. The most common sinonasal structures involved were the maxillary sinus $(61 \%)$, followed by the ethmoid sinus (29\%), nasal cavity/septum (26\%), sphenoid sinus (19\%) and two cases that involved the frontal sinus (6\%). Sinonasal location of involvement as well as other organs involved are shown in Table 1. Because sinonasal IgG4-RD can often present exclusively within the sinuses, it is important for rhinologists and general otolaryngologists to know when it is appropriate to consider this entity among the differential diagnosis.

\section{Epidemiology}

IgG4-RD has been shown to be most prevalent in the older male population with a reported male to female ratio of 1.6-4:1, ${ }^{26-28}$ and the most recent review of sinonasal IgG4-RD by Song et al. ${ }^{6}$ was consistent with that. However, this review demonstrates a nearly 1:1 ratio between males and females (16:15, or 53\% male). Interestingly, Mulholland et al. ${ }^{4}$ also reported a ratio of nearly 1:1 for all head and neck involvement. Additionally, the average age of diagnosis for IgG4-RD has been reported to be in the $6^{\text {th }}$ decade of life, but the review of cases with sinonasal involvement presented here demonstrates a younger average age of presentation of 46 years, with a few cases of teenage patients as young as 13, as summarized in Table 2.

\section{Presenting symptoms}

Common presenting symptoms of sinonasal IgG4-RD from this article's review include nasal obstruction/congestion (48\%), facial swelling (26\%), headache (19\%), and epistaxis or blood-tinged rhinorrhea $(16 \%)$. While only five other cases $(16 \%)$ presented a multiple-year history of chronic rhinosinusitis like this patient, IgG4-RD might be an important diagnostic consideration in patients with intractable CRS, especially when imaging is suggestive of the disease. Additionally, a few other cases described recurrent facial pain, congestion, and nasal discharge that were consistent with CRS in whom this diagnosis was not made. These presenting symptoms are, therefore, important to consider when making the diagnosis of sinonasal IgG4-RD. Presenting symptoms are listed in Table 3.

For the patient in the present case, sinonasal endoscopy was performed at each of the clinic appointments during the nine years he was managed within this institution. The Lund-Kennedy (LK) endoscopic scoring system is a standardized method of quantifying the visual appearance of the sinuses on nasal endoscopy ${ }^{29}$. The reliability of the system is improved by excluding the subscores of scarring and crusting, and correlate more closely to patient-reported outcome measures such as the SNOT-22 in both unoperated and postoperative patients. ${ }^{29}$ Table 4 denotes the date of assessment, LK score for the left and right nasal cavity, as well 
as the isolated bacteria and culture-directed antibiotics that were used to treat the patient.

\section{Imaging Characteristics}

The majority of cases demonstrated thickening, attenuation, or enhancement of the soft tissue in the sinuses with or without a discreet mass. However, Ueno et al. ${ }^{21}$ describes 5 cases where imaging revealed only fluid retention in the involved sinuses. Importantly, bony destruction or erosion was reported in the imaging description of $13(45 \%)$ of these cases. It is possible that additional cases had this feature on imaging but did not report it. Because bony erosion or tissue destruction might lead a clinician to suspect malignancy, it is important to rule this out histologically. In the present case, there was soft tissue enhancement without a discrete mass or bony destruction.

\section{Immunochemistry and Histopathology}

Definitive international pathology consensus guidelines for diagnosis were formally described in 2012 by Deshpande et al. ${ }^{2}$ Because serum IgG4 levels are neither sensitive nor specific for IgG4-RD, histopathology is required to confirm the diagnosis. The hallmark histologic findings for IgG4-RD include lymphoplasmacytic infiltration, storiform fibrosis, obliterative phlebitis, and mild to moderate tissue eosinophilia. While the exact findings and numbers of IgG4-positive cells vary depending on the tissue affected, the diagnosis of IgG4-RD is based primarily on IgG4-positive to IgG-containing cell ratio and the number of IgG4 positive cells per high powered field. A ratio of IgG4 to total IgG $>40 \%$ or IgG4 positive cells per high-power field $>10$ is considered to be diagnostic of IgG4-RD. While serum IgG4 levels are not diagnostic alone, levels $>135 \mathrm{mg} / \mathrm{dL}$ is also suggestive of IgG4-RD and might correlate with the severity of disease. ${ }^{2,} 30$

Elevated serum levels of IgG4 are usually reported in $70-80 \%$ of all patients with IgG4-RD, ${ }^{31,} 32$ although the results of a study by Carruthers et al. ${ }^{30}$ found that the sensitivity for detecting disease with a serum IgG4 levels $>135 \mathrm{mg} / \mathrm{dL}$ might actually be as high as $90 \%$. The present review of sinonasal IgG4-RD is consistent with these findings with elevated serum levels in $75 \%$ of cases $(\mathrm{N}=28)$. All of the cases that reported a value of IgG4 + plasma cells/HPF (19 cases) $>10$, most of which were $>30$ (79\%), although several others reported "diffuse" or "many" IgG4+ plasma cells instead of numeric values. All of the cases that reported IgG4 + cells/total IgG containing cells ratio (16 cases) had $>40 \%$. Laboratory and histologic summary from the present review is detailed in Table 5 .

\section{Treatment}

There are many approaches to treatment of IgG4-RD which usually involve some combination of surgical resection of affected tissues, systemic glucocorticoids, steroid-sparing immunosuppressive drugs and biological agents. Prednisone monotherapy at a dose of $0.6 \mathrm{mg} / \mathrm{kg} /$ day and tapered over the period of 3-6 months is currently considered the first-line therapy. ${ }^{26}$

In a recent study, the recurrence rate for IgG4-RD was reported to be $46 \%$ after initial treatment with steroids. ${ }^{26}$ However, this review of sinonasal disease demonstrated that, of the 14 patients who underwent steroid monotherapy initially, only 1 had recurrent disease (7\%). In that case, the addition of rituximab led to clinical improvement. Of the 2 patients who only underwent surgical resection anddebulking for whom follow-up was reported, both developed disease recurrence. However, Inoue et al. ${ }^{15}$ described one patient with sinusitis and no notable mass lesions who underwent sinus surgery alone, and showed no evidence of recurrence after a year. Of the 5 patients who had both surgery and steroid treatment, 1 had recurrent disease $(20 \%)$ which improved after the addition of rituximab. While one case presented by Kaur et al. ${ }^{24}$ reported no recurrence with the use of rituximab alone, that was not true of the case presented here. While the patient did have initial improvement with his initial two infusions of rituximab in 2012, his disease recurred a year later and persisted despite a third infusion of rituximab and intermittent oral steroids. However, after additional surgical resection of the affected maxillary sinus with mega-antrostomy and steroid rinses, the patient has been symptom-free and without evidence of clinical recurrence for 5 years. Treatment and follow-up outcomes from the cases in this article's review are described in Table 6 .

\section{CONCLUSION}


While a greater sample size is required to draw definitive conclusions, the findings presented here, which include the most comprehensive review of literature to date, suggest that the general otolaryngologist or rhinologist should have a high index of suspicion for this disease process when considering patients who present with new-onset symptoms of chronic sinusitis, particularly middle-aged adults without a longstanding history of sinonasal complaints or patients with refractory disease despite appropriate surgical intervention. When sinonasal IgG4-RD is considered, the authors of this article recommend diagnostic workup that includes CT sinuses without contrast, serum IgG4, and biopsy of the involved sinus tissue, if feasible. Although the utility of PET-CT in the diagnosis of IgG4-RD has been supported, sinonasal IgG4-RD is often limited to the head and neck and therefore it should not be used for the initial diagnostic workup, however, it can be considered once the diagnosis has been confirmed in order to identify other organ involvement or to monitor treatment response. ${ }^{33}$

Additionally, these findings suggest that steroid monotherapy is usually sufficient to prevent recurrence in sinonasal IgG4-RD. Although surgery may be indicated to improve symptoms, caution may be warranted, since it does not seem to reduce the likelihood of recurrence. The findings of this case and literature review also confirm that rituximab is an important consideration with recurrent disease after steroid monotherapy. A summary of diagnostic workup recommendations by the authors of this report can be found in Table 7 .

\section{REFERENCES}

1. Hamano H, Kawa S, Horiuchi A, et al. High Serum IgG4 Concentrations in Patients with Sclerosing Pancreatitis. New England Journal of Medicine . 2001;344(10):732-738. doi:10.1056/nejm200103083441005

2. Deshpande V, Zen Y, Chan JK, et al. Consensus statement on the pathology of IgG4-related disease. Modern Pathology . 2012;25(9):1181-1192. doi:10.1038/modpathol.2012.72

3. Takano K, Yamamoto M, Takahashi H, Himi T. Recent advances in knowledge regarding the head and neck manifestations of IgG4-related disease. Auris Nasus Larynx . 2017;44(1):7-17. doi:10.1016/j.anl.2016.10.011

4. Mulholland GB, Jeffery CC, Satija P, Côté DWJ. Immunoglobulin G4-related diseases in the head and neck: a systematic review. Journal of Otolaryngology - Head $\&$ Neck Surgery . 2015;44(1)doi:10.1186/s40463015-0071-9

5. Ishida M, Hotta M, Kushima R, Shibayama M, Shimizu T, Okabe H. Multiple IgG4-related sclerosing lesions in the maxillary sinus, parotid gland and nasal septum. Pathol Int . Sep 2009;59(9):670-5. doi:10.1111/j.1440-1827.2009.02425.x

6. Song BH, Baiyee D, Liang J. A Rare and Emerging Entity: Sinonasal IgG4-related Sclerosing Disease. Allergy \& Rhinology . 2015;6(3):ar.2015.6.0136. doi:10.2500/ar.2015.6.0136

7. Ikeda R, Awataguchi T, Shoji F, Oshima T. A case of paranasal sinus lesions in IgG4-related sclerosing disease. Otolaryngology-Head and Neck Surgery . 2010;142(3):458-459. doi:10.1016/j.otohns.2009.09.019

8. Sasaki T, Takahashi K, Mineta M, Fujita T, Aburano T. Immunoglobulin G4-Related Sclerosing Disease Mimicking Invasive Tumor in the Nasal Cavity and Paranasal Sinuses. American Journal of Neuroradiology . 2012;33(2):E19-E20. doi:10.3174/ajnr.a2495

9. Alt JA, Whitaker GT, Allan RW, Vaysberg M. Locally Destructive Skull Base Lesion: IgG4-related Sclerosing Disease. Allergy \&3 Rhinology . 2012;3(1):ar.2012.3.0026. doi:10.2500/ar.2012.3.0026

10. Lindau RH, Su YB, Kobayashi R, Smith RB. Immunoglobulin G4-related sclerosing disease of the paranasal sinus. Head Neck . Oct 2013;35(10):E321-4. doi:10.1002/hed.23175

11. Prabhu SM, Yadav V, Irodi A, Mani S, Varghese AM. IgG4-related disease with sinonasal involvement: A case series. Indian J Radiol Imaging . Apr 2014;24(2):117-20. doi:10.4103/0971-3026.134384

12. Morris C, Ng T, Kevin P, Singh N. Immunoglobulin G4 related disease isolated to the nasal cavity: a rare cause of nasal obstruction. The Journal of Laryngology \& Otology . 2015;129(S1):S57-S59. 
doi:10.1017/s0022215114001911

13. Kurien R, Babu TR, Rupa V. Unusual cause of maxillary sinus mass with proptosis. BMJ Case Reports . 2015:bcr2015210627. doi:10.1136/bcr-2015-210627

14. Kojima M, Nakazato Y, Hirabayashi K, Masawa N, Nakamura N. IgG4-related plasma cell granuloma of the maxillary sinus: A report of 2 cases. Ear Nose Throat J . Sep 2015;94(9):E17-9.

15. Inoue A, Wada K, Matsuura K, et al. IgG4-related disease in the sinonasal cavity accompanied by intranasal structure loss. Auris Nasus Larynx . Feb 2016;43(1):100-4. doi:10.1016/j.anl.2015.05.005

16. Chen B-N. IgG4-related disease presenting with destructive sinonasal lesion mimicking malignancy. European Archives of Oto-Rhino-Laryngology . 2016;273(11):4027-4029. doi:10.1007/s00405-016-4033-2

17. Vandjelovic ND, Humphreys IM. Immunoglobulin G4-related Sclerosing Disease of the Paranasal Sinuses: A Case Report and Literature Review.Allergy \& Rhinology . 2016;7(2):ar.2016.7.0154. doi:10.2500/ar.2016.7.0154

18. Gontarz M, Wyszyńska-Pawelec G, Zapała J, Gałązka K, Tomaszewska R, Lazar A. IgG4-related disease in the head and neck region: report of two cases and review of the literature. Polish Journal of Pathology . 2016;4:370-375. doi:10.5114/pjp.2016.65871

19. Bashyam A, Nagala S, Tahir F, Mirza S. Immunoglobulin G4-related disease of the paranasal sinuses. BMJ Case Reports . 2018:bcr-2018-224472. doi:10.1136/bcr-2018-224472

20. Chowsilpa S, Chowsilpa S, Teeranoraseth T, Roongrotwattanasiri K. Temporal bone involvement of IgG4-related disease: a rare condition misleading to petrous apicitis causing lateral rectus palsy. BMJ Case Reports . 2019;12(2):e228550. doi:10.1136/bcr-2018-228550

21. Ueno M, Nakano K, Miyagawa I, Tanaka Y. Five Cases of IgG4-related Disease with Nasal Mucosa and Sinus Involvement. Internal Medicine . 2020;59(15):1905-1911. doi:10.2169/internalmedicine.4127-19

22. Jurkov M, Olze H, Klauschen F, Bertelmann E, Schneider U, Arens P. IgG4-related orbitopathy as an important differential diagnosis of advanced silent sinus syndrome. HNO . 2020;68(S1):65-68. doi:10.1007/s00106-019-00799-8

23. Kouwenberg WL, Dieleman FJ, Willems SM, Rosenberg A. Inflammatory pseudotumour of the alveolar process of the maxilla as clinical manifestation of IgG4-related disease: a case report and literature review. Int J Oral Maxillofac Surg . Jun 2020;49(6):722-725. doi:10.1016/j.ijom.2019.11.008

24. Kaur K, Kakkar A, Manchanda S, et al. Sinonasal IgG4-related disease: a rare and emerging entity broadening the differential diagnosis in the sinonasal universe. European Archives of Oto-Rhino-Laryngology . 2021;doi:10.1007/s00405-020-06564-w

25. Hu EK, Parrish C, Wrobel B, Deshpande V, Stone JH. Immunoglobulin G4-related disease presenting as an ethmoid and maxillary mass.Ann Allergy Asthma Immunol . Jul 2013;111(1):75-7. doi:10.1016/j.anai.2013.05.007

26. Karadeniz H, Vaglio A. IgG4-related disease: a contemporary review. Turk J Med Sci . Nov 3 2020;50(SI2):1616-1631. doi:10.3906/sag-2006-375

27. Stone JH, Zen Y, Deshpande V. IgG4-Related Disease. New England Journal of Medicine . 2012;366(6):539-551. doi:10.1056/nejmra1104650

28. Mahajan VS, Mattoo H, Deshpande V, Pillai SS, Stone JH. IgG4-related disease. Annu Rev Pathol . 2014;9:315-47. doi:10.1146/annurev-pathol-012513-104708

29. Psaltis AJ, Li G, Vaezeafshar R, Cho KS, Hwang PH. Modification of the Lund-Kennedy endoscopic scoring system improves its reliability and correlation with patient-reported outcome measures. Laryngoscope . Oct 2014;124(10):2216-23. doi:10.1002/lary.24654 
30. Carruthers MN, Khosroshahi A, Augustin T, Deshpande V, Stone JH. The diagnostic utility of serum IgG4 concentrations in IgG4-related disease.Annals of the Rheumatic Diseases . 2015;74(1):14-18. doi:10.1136/annrheumdis-2013-204907

31. Nambiar S, Oliver TI. IgG4 Related Disease. StatPearls . 2020.

32. Ghazale A, Chari ST, Smyrk TC, et al. Value of serum IgG4 in the diagnosis of autoimmune pancreatitis and in distinguishing it from pancreatic cancer. Am J Gastroenterol . Aug 2007;102(8):1646-53. doi:10.1111/j.1572-0241.2007.01264.x

33. Zhao Z, Wang Y, Guan Z, Jin J, Huang F, Zhu J. Utility of FDG-PET/CT in the diagnosis of IgG4related diseases. Clin Exp Rheumatol . Jan-Feb 2016;34(1):119-25.

\section{Hosted file}

Table 1. Lit Review Info_Final.docx available at https://authorea.com/users/435464/articles/ 538468-sinonasal-igg4-rd-case-report-and-systematic-review

\section{Hosted file}

Table 2. Demographics.docx available at https://authorea.com/users/435464/articles/538468sinonasal-igg4-rd-case-report-and-systematic-review

\section{Hosted file}

Table 3. Presenting Symptoms.docx available at https://authorea.com/users/435464/articles/ 538468-sinonasal-igg4-rd-case-report-and-systematic-review

\section{Hosted file}

Table 4. Lund Kennedy.docx available at https://authorea.com/users/435464/articles/538468sinonasal-igg4-rd-case-report-and-systematic-review

\section{Hosted file}

Table 5. Lab Findings.docx available at https://authorea.com/users/435464/articles/538468sinonasal-igg4-rd-case-report-and-systematic-review

\section{Hosted file}

Table 6. Treatment.docx available at https://authorea.com/users/435464/articles/538468sinonasal-igg4-rd-case-report-and-systematic-review

\section{Hosted file}

Table 7. Workup.docx available at https://authorea.com/users/435464/articles/538468sinonasal-igg4-rd-case-report-and-systematic-review 